\title{
Quantum complete prelude to inflation
}

\author{
Stefan Hofmann, ${ }^{1, *}$ Marc Schneider, ${ }^{2, \dagger}$ and Maximilian Urban ${ }^{1, \$}$ \\ ${ }^{1}$ Arnold Sommerfeld Center for Theoretical Physics, Theresienstraße 37, 80333 München, Germany \\ ${ }^{2}$ Institute for Gravitation and the Cosmos, University Park, 16802-6300 Pennsylvania, USA
}

(Received 14 January 2019; published 22 March 2019)

\begin{abstract}
It is shown that the inflationary paradigm admits quantum complete extensions of spacetime. The extended inflationary spacetimes still have geodesic borders, but quantum fields are prohibited from migrating across these borders by their evolution semigroups. The geodesic singularities lurking across the borders lack a physical description because the evolution semigroups give vanishing probabilistic support to quantum fields for populating regions bordering on these singularities. As an example, anisotropic Bianchi type-I cosmologies are shown to be quantum complete preludes to inflation. They admit Kasnerlike geometries close to their geodesic borders. Quantum fields enjoy a contractive evolution in these asymptotic regions and ultimately become free. As a consequence, quantum probes cannot migrate across the geodesic border of Bianchi type-I cosmologies.
\end{abstract}

DOI: 10.1103/PhysRevD.99.065012

\section{INTRODUCTION}

A proper inflationary epoch in the primordial expansion history allows us to relate the variety of cosmic structures to quantum fluctuations of the dominant source during this stage [1-3]. These fluctuations represent adiabatic deviations from local isotropy and constitute the primordial seeds of gravitationally driven structure formation. As the microscopic origin of large-scale cosmic structures, inflation has passed all observational stress tests.

Inflation extends the cosmic history prior to a decelerated Friedmann cosmology with an accelerated expansion stage, which is past-directed timelike and null geodesic incomplete and which initially borders on a spacelike singularity. However, inflation can emerge from spatially anisotropic but homogeneous cosmologies as described by Bianchi type-I spacetimes. These cosmologies develop forward instabilities towards approximate isotropic spaces in inflating spacetime regions [4]. In addition, Kasner spacetimes develop backward instabilities triggering Bianchi type-II like transitions [5]. between different anisotropic cosmologies, leading to successions of Kasner geometries [6]. Kasner spacetimes are past-directed timelike and null geodesic incomplete as well. They have been

\footnotetext{
*stefan.hofmann@physik.uni-muenchen.de †mms94@psu.edu

\#maximilian.urban@physik.uni-muenchen.de
}

Published by the American Physical Society under the terms of the Creative Commons Attribution 4.0 International license. Further distribution of this work must maintain attribution to the author(s) and the published article's title, journal citation, and DOI. Funded by SCOAP. shown to be singularity free in canonical quantum cosmology [7].

If geodesic boundaries can be probed in measurement processes, they are manifest in observables and imply a physical pathology, since then observers can be located at the geodesic borders. For instance, the singular coincidence limit of Newton's gravitational potential field is not sufficient to imply a physical pathology. A sufficient condition is the existence of initial conditions allowing us to realize this coincidence limit dynamically in a finite time. Moreover, a repulsive Coulomb potential field is also singular in the coincidence limit, but since it is unbounded from above near zero, this limit cannot be realized dynamically. The Coulomb potential is complete near zero for repulsive charge configurations but incomplete for attractive charge configurations. However, even for attractive charge configurations, the Coulomb potential can still give rise to a complete quantum evolution, as it does for electrons in hydrogen bound states.

Whether a potential allows for a complete evolution is a context-sensitive question. A similar statement does not hold for spacetimes: It is meaningless to conclude that a spacetime supports an incomplete classical evolution but a complete quantum evolution. If both conclusions are valid, then the spacetime is simply incomplete. The distinction between classical and quantum evolution is still important because geodesic completeness might fail as a sound physical concept, while it remains a valid geometrical characterization of the mathematical spacetime model. As a physical concept, geodesic completion refers to observations and operational instructions for measurements based on classical mechanics.

In its simplest realization, a measurement process involves three dynamical subsystems: A system on which 
observers wish to perform measurements using specific apparatus in a given spacetime. Usually, it is assumed that the inertia of the apparatus is large compared with that of the system. This implies that during the measurement there is no significant backreaction of the system on the apparatus. In addition, it is assumed that the spacetime and its evolution is not affected by the experiment. To guarantee the converse, the spacetime region supporting the measurements is required to be sufficiently homogeneous. Close to the geodesic border of Kasner spacetimes, the latter requirement fails to hold for mechanical devices. Tidal forces become too strong during finite time measurements for these devices to operate as external apparatus. The same is true for recording devices of finite extent with mechanically interconnected circuit elements.

This leads us to incorporate measurement processes as internal phenomena with no external aspects. The system to be examined consists of field configurations and the only sensible bookkeeping devices are spectator fields which are locally coupled to these field configurations. During a measurement all field configurations might be significantly altered by spacetime variations. The backreaction of all field configurations on spacetime is still required to be negligible. In this respect, the singularity theorems [8] and our results concerning quantum regularity share the same postulate. For the sake of keeping the Introduction nontechnical, this common postulate can be stated informally as follows: The inertia of spacetime is large compared to that of all field configurations. In other words, the spacetime is effectively decoupled from all field configurations that compose the system and the observers. This decoupling postulate can be (in)validated a posteriori.

The ground state of the examined field configurations is time-dependent in dynamical spacetimes. In general, this time dependence is not given by a phase factor, i.e., the evolution of quantum states is not given by a unitary group. One can show that even in trivial cases unitarity fails to hold [9]. Here quantum states refer to wave functionals over the configuration space in the Schrödinger representation of quantum field theory. Instead, the evolution is naturally given by a semigroup, because the background is assumed to be absolutely inert against quantum fluctuations. A probabilistic interpretation is still possible, provided the semigroup enjoys a contraction property that replaces the ordinary isometry requirement. Physically the contraction property accommodates damping phenomena in the Schrödinger Hamiltonian which reduce the normalization of the wave functional. This implies that the Schrödinger Hamiltonian is not selfadjoint in the physical state space. Effectively dynamical spacetimes can act as damping media.

In contrast to a preinflationary Kasner phase, a pure de Sitter phase is subjected to a divergent time-evolution towards the initial singularity. The coincidence limit renders this space-time quantum incomplete. Without imposing an initial boundary condition at the time of Big Bang, finite amplitude tensor fluctuations at finite times will be amplified to trans-Planckian values when evolved backwards [10]. The failure of the no boundary proposal imposes a general obstruction to de Sitter cosmology close to the geodesic border and serves as a plea for a preinflationary phase.

In this article, we show that generic quantum inflaton fields cannot migrate across the geodesic boundary of Bianchi type-I cosmologies. This is guaranteed by a contractive evolution semigroup that assigns a vanishing probability for inflaton fields to populate the geodesic border. As a consequence, the spacelike singularity lurking beyond this border is decoupled from physical spacetime. The result is universal in the sense that it holds for any inflaton potential. This universality can be interpreted as a consequence of the Belinskii-Khalatnikhov-Lifshitz conjecture [11,12] which is operative at the geodesic border and guarantees that the dynamics at any spatial point is pefectly captured by an ordinary differential equation [13].

\section{QUANTUM COMPLETENESS}

Consider a globally hyperbolic spacetime $(\mathcal{M}, g)$ and choose a time function $t$ and a vector field $v$ on $\mathcal{M}$ such that the surfaces $\left(\Sigma_{t}\right)_{t \in \mathcal{I} \subset \mathbb{R}}$ of constant time are Cauchy hypersurfaces and such that $\nabla_{v} t=-1$. Note that the time interval $\mathcal{I}$ might be the entire real line, but we are mostly concerned with the time interval $\left.\mathcal{I}:=] 0, t_{\text {in }}\right]$. We denote by $\mathcal{C}\left(\Sigma_{t}\right)$ the configuration space of instantaneous field configurations $\phi$ on $\Sigma_{t}$, which is infinite-dimensional. For simplicity, $\phi: \mathcal{M} \rightarrow \mathbb{K}$, where $\mathbb{K}=\{\mathbb{R}, \mathbb{C}\}$. Let $\left(\mathcal{C}\left(\Sigma_{t}\right), \mathcal{D} \phi\right)$ denote a formal measure space, and let $\mathcal{L}^{2}\left(\mathcal{C}\left(\Sigma_{t}\right)\right)$ denote the $\mathbb{C}$-vector space of wave functionals $\Psi_{t}: \mathcal{C}\left(\Sigma_{t}\right) \rightarrow \mathbb{C}$ which are measureable and whose modulus is square integrable with respect to the functional measure $\mathcal{D} \phi$. We introduce the usual seminorm on $\mathcal{L}^{2}\left(\mathcal{C}\left(\Sigma_{t}\right)\right)$ :

$$
\left\|\Psi_{t}\right\|_{2}:=\left\{\int_{\mathcal{C}\left(\Sigma_{t}\right)} \mathcal{D} \phi\left|\Psi_{t}[\phi]\right|^{2}\right\}^{1 / 2}
$$

In general, this is not a norm on $\mathcal{L}^{2}\left(\mathcal{C}\left(\Sigma_{t}\right)\right)$ since $\left\|\Psi_{t}\right\|_{2}=0$ only implies $\Psi_{t}[\phi]=0$ almost everywhere in $\mathcal{C}\left(\Sigma_{t}\right)$ but not $\Psi_{t} \equiv 0$. For this reason, we introduce

$$
\begin{gathered}
\mathcal{N}\left(\mathcal{C}\left(\Sigma_{t}\right), \mathcal{D} \phi\right):=\left\{\Psi_{t} \in \mathcal{L}^{2}\left(\mathcal{C}\left(\Sigma_{t}\right), \mathcal{D} \phi\right): \Psi_{t}[\phi]=0\right. \\
\mathcal{D} \phi-\text { almost everywhere }\}
\end{gathered}
$$

$\mathcal{N}\left(\mathcal{C}\left(\Sigma_{t}\right), \mathcal{D} \phi\right)$ is a subspace of $\mathcal{L}\left(\mathcal{C}\left(\Sigma_{t}\right), \mathcal{D} \phi\right)$, and $\|\cdot\|_{2}$ is a norm on the quotient space

$$
L^{2}\left(\mathcal{C}\left(\Sigma_{t}\right), \mathcal{D} \phi\right):=\mathcal{L}^{2}\left(\mathcal{C}\left(\Sigma_{t}\right), \mathcal{D} \phi\right) \backslash \mathcal{N}\left(\mathcal{C}\left(\Sigma_{t}\right), \mathcal{D} \phi\right)
$$


Note that $L^{2}\left(\mathcal{C}\left(\Sigma_{t}\right), \mathcal{D} \phi\right)$ is not a space of wave functionals, but a space of equivalence classes of wave functionals. All operations are defined with respect to representatives.

Concerning interpretation, $\left|\Psi_{t}[\phi]\right|^{2}$ is a probability density in the following sense: If $\mathcal{U}$ is a (measurable) subset of $\mathcal{C}\left(\Sigma_{t}\right)$ and $\mathcal{X}_{\mathcal{U}}$ its indicator functional, then $\left\|\mathcal{X}_{\mathcal{U}} \Psi_{t}\right\|_{2}{ }^{2}$ is the probability for the field configuration on $\Sigma_{t}$ to be given by some $\phi \in \mathcal{U}$. This interpretation requires $\left\|\Psi_{t}[\phi]\right\|_{2}=1$ or at least $\Psi_{t}$ to be a normalizable functional for $t \in \mathcal{I}$. The smeared configuration field operator $\Phi[f]$ is just the operator for multiplication with $\phi[f]$, where $f$ is a smooth smearing function of compact support in $\Sigma_{t}$ such that the expectation value $\left\langle\Psi_{t}|\Phi[f]| \Psi_{t}\right\rangle=\left\|\sqrt{|\phi[f]|} \Psi_{t}\right\|_{2}^{2}$ is well defined. As usual, a measure for the scatter of field configurations around the expectation value is given by $\left\|\left(\Phi[f]-\phi[f] \mathrm{id}_{L^{2}}\right) \Psi_{t}\right\|_{2}$. The momentum field operator $\Pi[f]$ is the functional derivative $-\mathrm{i}\left(\operatorname{det}\left(g_{\Sigma_{t}}\right)\right)^{-1 / 2} \delta / \delta \phi$ in the direction of a smooth function $f$ of compact support. Here $g_{\Sigma_{t}}$ denotes the pullback of the metric field $g$ to the hypersurface $\Sigma_{t}(t \in \mathcal{I})$. Heisenberg's fundamental uncertainty relation follows from $\left[\Phi\left[f_{1}\right], \Pi\left[f_{2}\right]\right] \Psi_{t}[\phi]=$ $\mathrm{i}\left\langle f_{1}, f_{2}\right\rangle \Psi_{t}[\phi]$, where $\langle\cdot, \cdot\rangle$ denotes the canonical scalar product for smearing functions on $\Sigma_{t}$. It should be stressed that the above construction is only meant at a formal level.

We now consider a family of formal evolution operators $\left\{\mathcal{E}_{g}\left(t, t_{0}\right): 0 \leq\left|t-t_{0}\right|<\infty\right\}$ on $L^{2}\left(\mathcal{C}\left(\Sigma_{t}\right), \mathcal{D} \phi\right)$. Such a family is a strongly continuous semigroup if $\mathcal{E}_{g}\left(t_{0}, t_{0}\right)=$ $\mathrm{id}_{L^{2}}, \mathcal{E}_{g}(s, t) \mathcal{E}_{g}\left(t, t_{0}\right)=\mathcal{E}_{g}\left(s, t_{0}\right)$ for all $\left.s, t, t_{0} \in\right] 0, \infty[$ with $s, t \leq t_{0}$ (which reflects our convention that the flow of time runs from $t_{0}$ towards 0 ), and if for each $\Psi_{t} \in$ $L^{2}\left(\mathcal{C}\left(\Sigma_{t}\right), \mathcal{D} \phi\right)$ the map $\mathcal{I} \subset \mathbb{R} \rightarrow L^{2}\left(\mathcal{C}\left(\Sigma_{t}\right), \mathcal{D} \phi\right)$, defined by $t \mapsto \mathcal{E}_{g}\left(t, t_{0}\right) \Psi$ is continuous. Such evolution semigroups arise naturally in the Schrödinger-picture quantum theory of fields in curved spacetimes. A probabilistic interpretation is only possible for a special class of evolution semigroups: A contractive evolution semigroup satisfies the additional requirement $\left\|\mathcal{E}_{g}\left(t, t_{0}\right)\right\| \leq 1$ for all $t \leq t_{0}$ in the time interval $\mathcal{I}$. Here the operator norm is defined as usual, $\left\|\mathcal{E}_{g}\left(t, t_{0}\right)\right\|:=\inf \left\{C \geq 0:\left\|\mathcal{E}_{g}\left(t, t_{0}\right) \Psi\right\|_{2} \leq\right.$ $C\|\Psi\|_{2}$ for all $\left.\Psi_{t} \in L^{2}\left(\mathcal{C}\left(\Sigma_{t}\right), \mathcal{D} \phi\right)\right\}$.

As in the case of unitary evolution groups, we obtain the generator of $\mathcal{E}_{g}\left(t, t_{0}\right)$ by differentiation with respect to $t$. Set $h_{g}(t):=\left(\operatorname{id}_{L^{2}}-\mathcal{E}_{g}\left(t, t_{0}\right)\right) /\left|t-t_{0}\right|$ and consider only $\Psi_{t} \in$ $L^{2}\left(\mathcal{C}\left(\Sigma_{t}\right), \mathcal{D} \phi\right)$ for which $h_{g}(t) \Psi_{t}$ exists in the limit $\left|t-t_{0}\right| \rightarrow 0$. We denote this limit by $h_{g}\left(t_{0}\right) \Psi_{t_{0}}$ and call $h_{g}\left(t_{0}\right)$ the infinitesimal generator of $\mathcal{E}_{g}\left(t, t_{0}\right)$. Of course, $h_{g}=H[\Phi, \Pi ; g]$, where $H$ denotes the Hamiltonian composed of configuration field operators $\Phi$ and conjugated momentum field operators $\Pi$ in $L^{2}\left(\mathcal{C}\left(\Sigma_{t}\right), \mathcal{D} \phi\right)$. For simplicity, we focus on a simple local quantum theory with a Hamiltonian density $\mathcal{H}=\mathcal{T}(\Pi ; g)+$ $\mathcal{V}(\Phi ; g)$. On the hypersurface $\Sigma_{t}$ the functional Laplacian is $\mathcal{T}:=\sqrt{g_{t t}} \Pi \circ \Pi / 2$, where $\mathcal{V}$ denotes the effective potential with $\sqrt{g_{t t}} g_{\Sigma_{t}}^{-1}(\mathrm{~d} \Phi, \mathrm{d} \Phi)$ included. The infinitesimal generator $h_{g}$ and the evolution operator $\mathcal{E}_{g}\left(t, t_{0}\right)$ are related as follows:

$$
\begin{gathered}
\mathcal{E}_{g}\left(t, t_{0}\right)=T_{\leftarrow} \exp \left\{-\mathrm{i} \int_{t_{0}}^{t} \mathrm{~d} t^{\prime} H[\Phi, \Pi ; g]\left(t^{\prime}\right)\right\} \\
H[\Phi, \Pi ; g]\left(t^{\prime}\right)=\int_{\Sigma_{t^{\prime}}} \mathrm{d} \mu \mathcal{H}\left(\Phi, \Pi ; g_{\Sigma_{t^{\prime}}}\right) .
\end{gathered}
$$

Here $\mathrm{d} \mu$ is the covariant measure on $\Sigma$ and $T_{\leftarrow}$ denotes the time-ordering starting at $t_{0}>t$.

We now turn to the crucial condition for the generator of an evolution semigroup. Consider a dual element $S \in$ $\left[L^{2}\left(\mathcal{C}\left(\Sigma_{t}\right), \mathcal{D} \phi\right)\right]^{*}$ which satisfies $\|S\|=\|\Psi\|_{2}$ and $S\left(\Psi_{t}\right)=$ $\left\|\Psi_{t}\right\|_{2}{ }^{2}$. We can think of $S$ as a normalized tangent functional to $\Psi_{t}$. The Hahn-Banach theorem guarantees that each wave functional $\Psi_{t} \in L^{2}\left(\mathcal{C}\left(\Sigma_{t}\right), \mathcal{D} \phi\right)$ has a normalized tangent functional. The generator $h_{g}$ of $\mathcal{E}\left(t, t_{0}\right)$ is called accretive if for each $\Psi_{t} \in L^{2}\left(\mathcal{C}\left(\Sigma_{t}\right), \mathcal{D} \phi\right)$ we have $\operatorname{Im}\left(S\left(h_{g} \Psi_{t}\right)\right) \leq 0$. The relation between contractive and accretive is almost straightforward: $h_{g}$ is the generator of a contractive evolution semigroup if and only if $h_{g}$ is accretive.

Suppose $\Sigma_{0}$ is a spacelike geodesic boundary of $(\mathcal{M}, g)$ located at $t \rightarrow 0$. We call $(\mathcal{M}, g)$ quantum complete if the evolution semigroup of wave functionals on test-field configurations is contractive in $(\mathcal{M}, g)$ and $\left\|\mathcal{E}\left(t, t_{0}\right)\right\| \rightarrow 0$ for $t \rightarrow 0$ [14]. This definition of quantum completeness tacitly assumes that the test-field configurations enjoy a healthy evolution in Minkowski spacetime.

\section{ANISOTROPIC PRELUDE}

The prelude to inflation considered in the work is given by an anisotropic Bianchi type-I cosmology with a sequence of Kasner spacetimes characterizing the neighborhood of its geodesic boundary. A Kasner-like preinflationary phase is consistent with observational bounds on the $e$-folds because of the fast developing forward instability [15]. Any spacetime $(\mathcal{M}, g)$ considered in this prelude is a multiple warped product manifold of the form $\mathcal{M}=\mathcal{I} \times_{w_{1}} \mathbb{R} \times_{w_{2}} \mathbb{R} \times_{w_{3}} \mathbb{R}$ furnished with a tensor field,

$$
g=-\pi^{*}(\mathrm{~d} t \otimes \mathrm{d} t)+\sum_{a=1}^{3}\left(w_{a} \circ \tau\right)^{2} \sigma_{a}^{*}\left(q_{a}\right),
$$

with positive warping functions $w_{a} \in C^{\infty}(\mathcal{I})$ $\left(a \in\{1,2,3\}\right.$ ). By $\tau$ and $\sigma_{a}$ we denote the projections onto the base (time interval) $\mathcal{I}$ and the fibers (in our case, one-dimensional subspaces), respectively, and $\pi^{*}$ and $\sigma_{a}{ }^{*}$ are the correponding pullbacks. The pairs $\left(\mathbb{R}, q_{a}\right)$ denote the flat Riemannian Fiber manifolds with respect to the base manifold $(\mathcal{I},-\mathrm{d} t \otimes \mathrm{d} t)$. Kasner spacetimes are multiple warped products of this type with warping functions $w_{a}=\mathrm{id}_{\mathcal{I}} p_{a}$, where $p_{a} \in \mathbb{R}$ denote the so-called Kasner exponents. The Kasner exponents are required to lie in the intersection of the Kasner plane $p_{1}+p_{2}+p_{3}=1$ and the Kasner sphere $p_{1}^{2}+p_{2}^{2}+p_{3}^{2}=1$. Kasner 
spacetimes can be characterized as Ricci-flat Einstein manifolds which are globally hyperbolic, future-directed timelike and null geodesic complete, but past-directed timelike and null geodesic incomplete. Since they are vacuum solutions, Kasner geometries can only be an approximate description close to the geodesic border of an anisotropic spacetime such as the more general Bianchi type-I models relevant for this work. Bianchi type-I geometries are multiple warped product manifolds $\mathcal{M}_{B}$ of the type (6).

Consider a classical scalar field $\phi: \mathcal{M}_{B} \rightarrow \mathbb{R}$ in a Bianchi type-I spacetime with Hamilton density $\mathcal{H}=\mathcal{T}(\pi ; g)+\mathcal{V}(\phi ; g)$. The effective potential $\mathcal{V}$ includes the inflaton potential [16]

$$
V=V_{\mathrm{dS}}\left(1-\exp \left(-\phi / \phi_{0}\right)\right)^{2} .
$$

This potential is harmonic in $\phi$ around its minimum and dominated by a spacetime homogeneous energy density $V_{\mathrm{dS}}$ away from it. The potential parameters are fixed as $\sqrt{G_{\mathrm{N}}} \phi_{0}=10^{-3}$ and $V_{\mathrm{dS}}=10^{13} \mathrm{GeV}$ in order to obtain the correct amplitude of metric perturbations in the $S O(3)$-scalar sector.

In the slow-roll regime, $\mathcal{T} \ll V_{\mathrm{dS}}$ at early times $t \ll 1 / H_{\mathrm{dS}}$ the anisotropic expansion history is given by a Kasner solution with warping functions $w_{a}(t) / w_{a}\left(t_{\text {in }}\right) \approx$ $\left(t / t_{\text {in }}\right)^{p_{a}}$ for $a \in\{1,2,3\}$. At these early times the approximate de Sitter source $H_{\mathrm{dS}}^{2}=(8 \pi / 3) G_{\mathrm{N}}\left(V_{\mathrm{dS}}+\mathcal{O}\left(\mathcal{K} / V_{\mathrm{dS}}\right)\right)$ is effectively decoupled from spacetime and Kasner geometries can emerge. At intermediate times $t \sim 1 / H_{\mathrm{dS}}$ the expansion history is given by a more general Bianchi type-I solution of Einstein's equation [17], $w_{a}(t) / \overline{w_{a}}=\left[\sinh \left(3 t H_{\mathrm{dS}}\right)\right]^{1 / 3}\left[\tanh \left(3 t H_{\mathrm{dS}} / 2\right)\right]^{p_{a}-1 / 3}$, where $\overline{w_{a}}$ denote normalization constants. While the exponents $p_{a}$ are in the intersection of the Kasner plane and Kasner sphere, the warping functions explicitly depend on the transition time $1 / H_{\mathrm{dS}}$. For late times, $t \gg 1 / H_{\mathrm{dS}}$ an approximate de Sitter stage emerges with $w_{a}(t) / w_{a}\left(t_{0}\right) \approx$ $\exp \left(\left(t-t_{0}\right) H_{\mathrm{dS}}\right)$.

Clearly, $1 / H_{\mathrm{dS}}$ is the characteristic time scale for isotropization driven by $V_{\mathrm{dS}}$ : Consider the Weyl tensor as a tensor of type $(0,4)$ given by $C=R-\frac{1}{2}\left(\mathrm{Ric}-\frac{1}{4} S g\right) *$ $g-\frac{1}{24} S g * g$, where $R$ denotes the type- $(0,4)$ Riemann tensor, Ric is the Ricci tensor and $S$ the curvature scalar. For any symmetric tensors $T_{1}, T_{2}$ of type $(0,2), T_{1} * T_{2}$ denotes the Kulkarni-Nomizu product. Throughout the evolution up to the end of inflation, Ric $\approx \frac{1}{4} S g$, so $C \approx R-\frac{1}{24} S g * g$. The Ricci decomposition is, therefore, $|R|^{2} \approx|C|^{2}+\left|\frac{1}{24} S g * g\right|^{2}$. At early times $t \ll 1 / H_{\mathrm{dS}}$ this decomposition into irreducible components with respect to the orthogonal group is dominated by the anisotropic contribution, $|R|^{2} \approx|C|^{2}=\left|p_{1} p_{2} p_{3}\right|(2 / t)^{4}$. The asymptotic behavior of the conformal tensor renders any de Sitter-like source initially irrelevant, which is why the
Kasner solution is a good description of the geometry in the vicinity of the cosmic singularity. In contrast, at late times $t \gg 1 / H_{\mathrm{dS}}$ the Ricci decomposition is dominated by the approximate de Sitter source $|R|^{2} \approx\left|\frac{1}{24} S g * g\right|^{2}$ because $|C|^{2} \approx \exp \left(-6 t H_{\mathrm{dS}}\right)$. In this stage, the approximate de Sitter source damps all anisotropic contributions to the curvature.

\section{KASNER SPACETIMES ARE QUANTUM COMPLETE}

The last section was devoted to geometric preliminaries of anisotropic preludes to inflation. These are given by approximate Kasner spacetimes which exhibit a forward instability towards more general Bianchi type-I cosmologies that allow for isotropization at a later stage. Kasner spacetimes border on singular hypersurfaces $\Sigma_{0}$ as well. This raises the question of how their geodesic boundary compares to the geodesic incompleteness of isotropic cosmologies. At the intersection of the Kasner plane and sphere $p_{a} \in\left[-1 / 3,1\right.$, excluding the case $p_{a}=0$ for some $a \in\{1,2,3\}$ which is isometric to an accelerated frame in Minkowski spacetime. The most interesting case is given by $p_{a}=-1 / 3$ for some Kasner index, then the remaining two exponents are equal to $2 / 3$. For definiteness and without loss of generality, consider $p_{1}=-1 / 3$, $p_{2}=p_{3}=2 / 3$. This is the unique solution with $p_{2}=p_{3}$. In general, the above half-closed interval for the Kasner exponents forces us to restrict the base (time interval) to $\left.\mathcal{I}=] 0, t_{\text {in }}\right]$, where for the upper limit $t_{\text {in }} \ll 1 / H_{\mathrm{dS}}$. Of course, for the exact Bianchi type-I solution $\mathcal{I}=] 0, \infty[$, but our focus is on the vicinity of the geodesic border $\Sigma_{0}$. Consider two spacetime events $P=\left(d_{\text {in }}, 0,0\right)$ and $Q=(0,0,0)$ relative to some coordinate neighborhood in the hypersurface $\Sigma_{\text {in }}$. Towards the geodesic border $\Sigma_{0}$ the proper spatial distance $d_{\text {phys }}$ between these events scales as $d_{\text {phys }}(t) / d_{\text {in }}=\left(t_{\text {in }} / t\right)^{2 / 3}$. Coincidence limits of events towards the singularity are prohibited by the Kasner conditions, while Friedmann cosmologies enforce coincidence limits, no matter how they are regularized in interacting field theories. This observation gives rise to the hypothesis that Kasner spacetimes are quantum complete in the sense of Sec. II.

Consider the space $\mathcal{C}\left(\Sigma_{t}\right)(t \in \mathcal{I})$ of instantaneous inflaton field configurations on the hypersurface $\Sigma_{t}$. We introduce a bilinear functional $\mathcal{K}_{t}: \mathcal{C}\left(\Sigma_{t}\right) \times \mathcal{C}\left(\Sigma_{t}\right) \rightarrow \mathbb{C}$, $\left(\phi_{1}, \phi_{2}\right) \rightarrow\left[\phi_{1}\right] \mathcal{K}_{t}\left[\phi_{2}\right]$ with coordinate representation

$$
\left[\phi_{1}\right] \mathcal{K}_{t}\left[\phi_{2}\right]=\int_{\Sigma_{t}} \mathrm{~d} \mu(x) \mathrm{d} \mu(y) \phi_{1}(x) K_{t}(x, y) \phi_{2}(y),
$$

where $\mathrm{d} \mu(x)=\sqrt{\operatorname{det}(q)} \mathrm{d}^{3} x$ and $q(t)$ denotes the spatial metric field induced on $\Sigma_{t}$. The bilocal kernel function $K$ is spatially homogeneous and the bilinear functional $\mathcal{K}$ is symmetric. In addition to spatial homogeneity, the scaling of $d_{\text {phys }}(t) / d_{\text {in }}=\left(t_{\text {in }} / t\right)^{2 / 3}$ implies that initially separated 
events remain uncorrelated close to the geodesic border, as is shown below. Events can only be correlated if the spacetime regions supporting them intersect. Before calculating the kernel, we may therefore guess that $K(x, y ; t)=k(t) \delta(x-y) / \sqrt{\operatorname{det}(q)(t)}$ in the vicinity of the geodesic singularity. It follows that

$$
\left[\phi_{1}\right] \mathcal{K}_{t}\left[\phi_{2}\right] \rightarrow k(t) \int_{\Sigma_{t}} \mathrm{~d} \mu(x) \phi_{1}(x) \phi_{2}(x)
$$

for $t \rightarrow 0$. If we specialize to $[\phi] \mathcal{K}_{t}[\phi]$ then the bilinear functional becomes a quadratic functional $\mathcal{K}_{t}[\phi]$ in this limit. Since the inflaton field experiences exclusively ultralocal self-correlations, a regularization prescription is required. Note that towards the geodesic border $\mathcal{K}_{t}[\phi]$ is similar to a time-dependent mass term in the Lagrange function for the inflaton field.

In the following, we consider smooth functions on $\Sigma_{t}$ and linear functionals on the algebra of these smooth functions, but our notation will not distinguish between them. The bilinear functional $\mathcal{K}_{t}$ can be described by on-shell configuration fields in $\mathcal{S}=\left\{\varphi: \mathcal{I} \times \Sigma_{t \in \mathcal{I}} \rightarrow\right.$ $\left.\mathbb{C}:\left(\square-m^{2}\right) \varphi=0\right\}$. Huygens's principle relates the kernel functional with on-shell configuration fields as follows

$$
J\left[-\mathrm{i} \partial_{t} \varphi\right]=[J] \mathcal{K}_{t}[\varphi]
$$

for any smooth detector source $J$. A more direct relation holds in Fourier space. Our convention for the Fourier transform is

$$
\mathcal{F}_{t} f(k):=\int_{\Sigma_{t}} \mathrm{~d}^{3} x \exp (-\mathrm{i} k \cdot x) f(t, x)
$$

for any $f$ in $L^{1}\left(\Sigma_{t}\right)$ or Schwartz space. The argument of the plane wave is $k \cdot x:=k_{a} x^{a}$. For all $f$ in Schwartz space, the function $f$ can be recovered from its Fourier transform by the inversion formula

$$
\mathcal{F}^{-1} \hat{f}(x)=(2 \pi)^{-3} \int_{\mathbb{R}^{3}} \mathrm{~d}^{3} k \exp (\mathrm{i} k \cdot x) f(t, k),
$$

where $\mathrm{d}^{3} k:=\mathrm{d} k_{1} \wedge \mathrm{d} k_{2} \wedge \mathrm{d} k_{3}$. For smooth fields, (10) implies $-\mathrm{i} \partial_{t} \varphi=K_{t} \star \varphi$, with $K_{t} \star \varphi$ denoting the covariant convolution of the bilocal kernel function and the configuration field on $\Sigma_{t}$. Using $\mathcal{F}^{-1} \widehat{K_{t}} \star \mathcal{F}^{-1} \hat{\varphi}=\mathcal{F}^{-1} \widehat{K_{t}} \hat{\varphi}$, where the Fourier transforms are with respect to the convolution variable, and $\partial_{t} \mathcal{F}^{-1} \hat{\varphi}=\mathcal{F}^{-1} \partial_{t} \hat{\varphi}$, Huygens's principle (10) can be written as

$$
\frac{-\mathrm{i}}{\sqrt{\operatorname{det}(q)}} \partial_{t} \ln \frac{\hat{\varphi}(t, k)}{\hat{\varphi}(\tau, k)}=\hat{K}(t, k),
$$

where $\tau$ is an arbitrary reference time in $\left.\mathcal{I}=] 0, t_{\text {in }}\right]$.
On-shell field configurations are easily calculated in the vicinity of the geodesic border to Kasner spacetimes. The d'Alembert operator is $\square=\partial_{t}^{2}+t^{-1} \partial_{t}-t^{-2 p_{a}} \partial_{a}^{2}$ with the spatial index running in $a \in\{1,2,3\}$. The ansatz $\varphi(t, x)=T(t) R(x)$ gives an ordninary second-order differential equation for $T$ with singular coefficients: $\left(\partial_{t}^{2}+t^{-1} \partial_{t}-\kappa^{\prime} t^{-2 \alpha} \mathrm{id}\right) T \approx 0$. Here $\alpha$ is the largest Kasner exponent, $\kappa^{\prime}$ is determined from the solution of $R$ and $\approx$ denotes equality up to irrelevant contributions, i.e., contributions which are less singular than those given. We introduce a pivotal time scale $t_{*}$ and $\varepsilon \tau:=t / t_{*}$, where $\varepsilon>0$ is a smallness parameter. Then $\left(\partial_{\tau}^{2}-\tau^{-1} \partial_{\tau}\right) T \approx$ $+\kappa \varepsilon^{2(1-\alpha)} \tau^{-2 \alpha} T$. Since $p_{a} \in[-1 / 3,1[$ the largest Kasner exponent is smaller than one. In the limit $\varepsilon \rightarrow 0$, the asymptotic solution is $T \approx c_{1}+c_{2} \ln (\varepsilon \tau)$ with $c_{1}$, $c_{2} \in \mathbb{R}$. If the Kasner spacetime is axisymmetric then explicit solutions are known in terms of Bessel and biconfluent Heun functions. Close to the geodesic border these agree with our asymptotic solution $\varphi(t, x)=C_{1}(x)+$ $C_{2}(x) \ln \left(t / t_{*}\right)$, where $C_{i}(i \in\{1,2\})$ are smooth complex valued functions on $\Sigma_{t}$ and $t, t_{*} \in \mathcal{I}$. For simplicity, we choose $t_{*}=t_{\text {in }}$ for the pivotal time scale such that $\varphi\left(t_{\text {in }}, x\right)=C_{1}(x), \dot{\varphi}\left(t_{\text {in }}, x\right)=C_{2}(x) / t_{\text {in }}$ and $\varphi(t, x)=$ $\varphi\left(t_{\text {in }}, x\right)-t_{\text {in }} \dot{\varphi}\left(t_{\text {in }}, x\right)\left|\ln \left(t / t_{\text {in }}\right)\right|$ for $\left.\left.t \in\right] 0, t_{\text {in }}\right]$. As a result, the kernel function is given by

$$
K_{t}(x, y) \approx-\frac{\mathrm{i}}{t^{2} \ln \left(t / t_{\text {in }}\right)}\left[\delta(x-y)-\frac{R(x, y)}{\ln \left(t / t_{\text {in }}\right)}\right] .
$$

Here $R:=\mathcal{F}^{-1} \widehat{C_{1}} / \widehat{C_{2}}$. To leading order in $\ln ^{-1}\left(t / t_{\text {in }}\right)$ $(t \in] 0, t_{\text {in }}[)$ the kernel function is given by a purely imaginary contact term. This contribution alone is a particular solution to Huygens's principle (10). It is universal in the following sense: Let us introduce a functional generalization of the Dirac measure $\delta: \mathcal{C}_{0}^{\infty}\left(\Sigma_{t}\right) \times \mathcal{C}_{0}^{\infty}\left(\Sigma_{t}\right) \rightarrow \mathbb{C}$, defined by $\left[f_{1}\right] \delta\left[f_{2}\right]:=f_{1}\left[f_{2}\right]$. Note that our notation does not distinguish between elements of $\mathcal{C}_{0}^{\infty}\left(\Sigma_{t}\right)$ and linear functionals on $\mathcal{C}_{0}^{\infty}\left(\Sigma_{t}\right)$. A particular kernel functional solving (10) is given by $\left[f_{1}\right] \mathcal{K}_{t}\left[f_{2}\right]=-\mathrm{i}\left[f_{1}\right] \delta\left[f_{2} \partial_{t} \ln \left(f_{2} / f_{2 \text { in }}\right)\right]$ can be extended to a bilinear functional associated with propagating waves. On its own it accounts only for the wave front at the location of the detector described by the current density $J$, but does not contain information about spatial correlations between wave fronts on $\Sigma_{t}$, which is partially contained in the bilinear functional $\mathcal{R}$ associated with $R$. This contribution, however, is already subleading in the vicinity of the geodesic border. Hence events at different spatial locations on $\Sigma_{t}$ are approximately uncorrelated close to the geodesic border. In other words, towards the geodesic singularity the spacetime probes consisting of quantum fields are reduced to decoupled pointlike degrees of freedom. The latter statement will be substantiated in the remainder of the article. 
Close to the classical equilibrium configuration of the inflaton potential (7) we make the following ansatz for the Schrödinger wave functional:

$$
\Psi_{t}[\phi]=\Psi_{t}^{(0)}[\phi] \times \exp \left(\mathcal{D}_{t}[\phi]\right) .
$$

Here, $\Psi_{t}^{(0)}$ denotes the ground state functional

$$
\begin{aligned}
\Psi_{t}^{(0)}[\phi] & =\mathcal{N}_{t}^{(0)} \mathcal{G}_{t}^{(0)}[\phi], \\
\mathcal{N}_{t}^{(0)} & =\mathcal{N}_{t_{\text {in }}}^{(0)} \exp \left\{+\frac{\mathrm{i}}{2} \int_{t_{\text {in }}}^{t} \mathrm{~d} \tau \int_{\Sigma_{\tau}} \mathrm{d} \mu \frac{1}{2} \Pi^{2}[\phi] \mathcal{K}_{\tau}[\phi]\right\}, \\
\mathcal{G}_{t}^{(0)}[\phi] & =\exp \left\{-\frac{1}{2}[\phi] \mathcal{K}_{t}[\phi]\right\}
\end{aligned}
$$

and $\mathcal{D}_{t}[\phi]$ generates non-Gaussian deformations of the ground state due to inflaton self-interactions caused by the potential (7). Note that $\mathcal{D}_{t}[\phi]=\sum_{n \geq 2} \mathcal{D}_{t}^{[n]}[\phi]$ is a sum of nonlinear functionals starting at quadratic order.

Consider the wave functional of the ground state in the vicinity of the geodesic border $\Sigma_{0}$. To leading and subleading order its normalization is given by

$$
\begin{aligned}
\frac{\mathcal{N}_{t}^{(0)}}{\mathcal{N}_{t_{\text {in }}}^{(0)} \approx} & \exp \left\{-\frac{1}{2} \operatorname{vol}_{\mathrm{ps}} \int_{t_{\mathrm{in}}}^{t} \frac{\mathrm{d} \tau}{\tau \ln \left(\tau / t_{\mathrm{in}}\right)}\right\} \\
& \times \exp \left\{+\frac{1}{2} R(0) \operatorname{vol}\left(\Sigma_{t}\right) \int_{t_{\mathrm{in}}}^{t} \frac{\mathrm{d} \tau}{\tau \ln ^{2}\left(\tau / t_{\mathrm{in}}\right)}\right\},
\end{aligned}
$$

where $\operatorname{vol}_{\mathrm{ps}}=\operatorname{vol}\left(\Sigma_{t}\right) \operatorname{vol}\left(T^{*} \Sigma_{t}\right)$ denotes the time-independent coordinate phase space volume, and $R(0)$ is the regularized value of the complex-valued bilocal function $R$ in the coincidence limit. As anticipated, the kernel function requires regularization in the spatial coincidence limit on any hypersurface $\left.\left.\Sigma_{t}(t \in] 0, t_{\text {in }}\right]\right)$. In particular, since $\mathrm{vol}_{\mathrm{ps}}$ is time-independent this requirement is logically independent from the existence of a geodesic border. Therefore it is sufficient to introduce crude cut-off regulators for the purposes of this article. We find

$$
\begin{aligned}
{\left[\ln \left(\mathcal{N}_{\tau}^{(0)}\right)\right]_{t_{\mathrm{in}}}^{t} \approx } & -\frac{\operatorname{vol}_{\mathrm{ps}}}{2}\left[\ln \left(\ln \left(\tau / t_{\mathrm{in}}\right)\right)\right]_{t_{\mathrm{in}}}^{t} \\
& -\frac{\operatorname{vol}\left(\Sigma_{t}\right)}{2} R(0)\left[\ln ^{-1}\left(\tau / t_{\mathrm{in}}\right)\right]_{t_{\mathrm{in}}}^{t} .
\end{aligned}
$$

For $t / t_{\text {in }} \rightarrow 0$, the ground state normalization $\mathcal{N}_{t}^{(0)} \propto$ $\exp \left(\Gamma_{t}\right)$ is exponentially suppressed with a damping factor $\Gamma_{t} \approx\left(-\operatorname{vol}_{\mathrm{ps}} / 2\right) \ln \left(\left|\ln \left(t / t_{\mathrm{in}}\right)\right|\right)$ up to an irrelevant phase in leading order. The real part of the damping factor monotonically approaches minus infinity when $t$ goes to zero.

In order to study $\mathcal{G}^{(0)}$, we decompose the bilocal kernel function $R=\operatorname{Re}(R)+\mathrm{i} \operatorname{Im}(R)$ and its associated bilinear functional $\mathcal{R}=\mathcal{R}_{\text {re }}+\mathrm{i} \mathcal{R}_{\text {im }}$ accordingly. Then,

$$
\begin{aligned}
\mathcal{G}_{t}^{(0)} \approx & \exp \left\{\frac{\mathrm{i}}{2} \frac{1}{t^{2} \ln \left(t / t_{\mathrm{in}}\right)}[\phi]\left(\delta-\frac{\mathcal{R}_{\mathrm{re}}}{\ln \left(t / t_{\text {in }}\right)}\right)[\phi]\right\} \\
& \times \exp \left\{\frac{1}{2} \frac{1}{t^{2} \ln ^{2}\left(t / t_{\text {in }}\right)}[\phi] \mathcal{R}_{\mathrm{im}}[\phi]\right\}
\end{aligned}
$$

towards the geodesic border $\Sigma_{0}$. Note that for any bilinear functional $\mathcal{A} \in\left\{\delta, \mathcal{R}_{\text {re }}, \mathcal{R}_{\text {im }}\right\}$ appearing in (19) the combination $t^{-2} \mathcal{A}$ is time independent. Therefore $\mathcal{G}^{(0)}[\phi] \rightarrow 1$ in the limit $t / t_{\text {in }} \rightarrow 0$ for any field configuration.

As a result, $\Psi_{t}^{(0)}[\phi] \rightarrow 0$ towards $\Sigma_{0}$, which implies

$$
\lim _{t \rightarrow 0}\left\|\Psi_{t}^{(0)}\right\|_{2}=0
$$

The ground state wave functional does not yield probabilistic support to any field configuration at the geodesic border. While Kasner spacetimes border at a geodesic singularity, they do not leak information across the geodesic border. There is no physical characterization of $\Sigma_{0}$ in terms of measurement processes and observables. By direct Kernel methods we have shown that $\left.\left.\left\{\mathcal{E}_{g}\left(t, t_{\text {in }}\right): t \in\right] 0, t_{\text {in }}\right]\right\}$ is a contraction semigroup describing the ground state evolution in asymptotic Kasner spacetimes. Since $\mathcal{E}_{g}\left(t, t_{\text {in }}\right)$ has an explicit kernel, we can qualify asymptotic Kasner geometries as quantum complete preludes to inflation (via intermediary Bianchi type-I cosmologies) by direct kernel methods. The succession from asymptotic Kasner geometries via Bianchi type-I to inflationary spacetimes is, therefore, a quantum complete sequence of physical spacetimes which is consistent with the results in [18]. The singular potential in anisotropic cosmologies has no effect on the consistency of scattering processes. In turn, extending inflation by an anisotropic prelude with asymptotic Kasner geometry results in a quantum complete inflationary paradigm.

Non-Gaussian deformations of the ground state are generated by self-interactions. We refrain from presenting the calculation of $\mathcal{D}_{t}$ here. The basic result is that selfinteractions do not alter the leading asymptotic behavior towards the geodesic border. Intuitively, this can be understood by direct kernel methods [19] as follows. The Hamilton density operator of the inflaton is $\mathcal{H}=\mathcal{T}(\Pi ; g)+$ $\mathcal{V}(\Phi ; g)$, where $\mathcal{T}=(\Pi)^{2} / 2=-1 /(2 \operatorname{det}(q)) \delta^{2} / \delta \phi^{2}$ denotes the functional Laplacian and $\mathcal{V}=q^{-1}(\mathrm{~d} \Phi, \mathrm{d} \Phi)+$ $V(\Phi)$ the effective potential density operator including the inflaton potential $V=V_{\mathrm{dS}}\left(\Phi / \phi_{0}\right)^{2}+V_{\mathrm{dS}} \mathcal{O}\left[\left(\Phi / \phi_{0}\right)^{3}\right]$ close to its minimum and $V=V_{\mathrm{dS}}$ away from it. Because the self-interactions are polynomial, expectation values of observables are moments of the probability density $\left|\Psi_{t}^{(0)}\right|^{2}$. It is convenient to add an auxiliary source term to the generating functional

$$
\mathcal{Z}_{t}[J]=\int_{\mathcal{C}\left(\Sigma_{t}\right)} \mathcal{D} \phi\left|\Psi_{t}^{(0)}[\phi]\right|^{2} \exp \left(\mathcal{J}_{t}[\phi]\right)
$$


with $J:=(1 / \sqrt{\operatorname{det}(q)(t)}) \delta \mathcal{J}_{t}[\phi] / \delta \phi$. In closed form,

$$
\mathcal{Z}_{t}[J]=\left|\mathcal{N}_{t}^{(0)}\right|^{2} \exp \left(\frac{1}{2}[J] \mathcal{K}_{t}^{-1}[J]\right),
$$

where $\mathcal{K}_{t}^{-1}$ is the covariant functional inverse of $\mathcal{K}_{t}$. It is easy to solve $\left[f_{1}\right]\left(K_{t} \star K_{t}^{-1}\right)\left[f_{2}\right]=\left[f_{1}\right] \delta\left[f_{2}\right]$ asymptotically. Close to the geodesic border

$$
\mathcal{K}_{t}^{-1} \approx \mathrm{i} t \ln \left(t / t_{\text {in }}\right)\left[\delta+\frac{\mathcal{R}}{\ln \left(t / t_{\text {in }}\right)}\right]
$$

up to order $\ln ^{-2}\left(t / t_{\text {in }}\right)$. Note that if the square bracket in (23) is replaced with an expression that is regular in the coincidence limit then $\mathcal{K}_{t}^{-1} \rightarrow 0$ for $t / t_{\text {in }} \rightarrow 0$. Close to the potential minimum and for $\left.t \in] 0, t_{\text {in }}\right]$

$$
\begin{aligned}
\left\langle\Psi_{t}^{(0)}|V(\Phi)| \Psi_{t}^{(0)}\right\rangle & \approx \\
& =V_{\mathrm{dS}}\left(\frac{1}{\phi_{0}} \frac{1}{\sqrt{\operatorname{det}(q)}} \frac{\delta}{\delta J}\right)^{2} \mathcal{Z}_{t}[0] \\
& \approx\left(V_{\mathrm{dS}} / \phi_{0}^{2}\right)\left|\mathcal{N}_{t_{\mathrm{in}}}^{(0)}\right|^{2} \exp \left(2 \Gamma_{t}\right) K_{t}^{-1}(x, x),
\end{aligned}
$$

where $K_{t}^{-1}(x, x)$ denotes the regularized bilocal kernel function corresponding to $\mathcal{K}_{t}^{-1}$ in the coincidence limit. This can be used as an anchor for an inductive procedure proving that self-interactions have vanishing probabilistic support towards the geodesic border of asymptotic Kasner geometries. Self-interactions respect the ground state. In this sense, Kasner geometries in the immediate vicinity of $\Sigma_{0}$ protect the ground state against non-Gaussian deformations, and in turn, the ground state renders these geometries quantum complete. This is a consequence of the BelinskiiKhalatnikov-Lifshitz conjecture which is at work here. At the operator level, effectively $\mathcal{H} \approx \mathcal{T}(\Pi ; g)$ close to the geodesic border.

\section{DISCUSSION AND CONCLUSION}

The main result of this article is the following: Kasner universes are quantum complete preludes to inflationary spacetimes that smoothly transit to ordinary Bianchi-type I cosmologies. They still have geodesic borders beyond which spacelike singularities are lurking. Nonetheless, these singularities do not constitute a physical pathology as such. For these singularities to present a physical pathology, they must admit a description based on observables and measurement processes. It is not sufficient to evaluate observables at the geodesic border, because it is a priori not clear whether the fields composing observables and measurement devices are supported at or close to the border. For an imperfect analogy: Newton's potential is singular in the coincidence limit. However, this is not sufficient to conclude that Newtonian gravity is plagued by physical pathologies. In order to promote the mathematical singularity to a physical pathology, initial conditions are required to exist such that the coincidence limit can dynamically be realized in a finite time. The same holds for spacetime singularities.

Certainly, curvature invariants are singular when evaluated at the geodesic borders of Bianchi type-I cosmologies. Again, the question is whether this evaluation corresponds to a physical measurement process. As an imperfect analog, consider the motion of a classical electric charge in a repulsive Coulomb potential on the half line $[0, \infty)$. Clearly the Coulomb potential has a mathematical singularity at the origin. However, any electrical charge with finite initial energy moving towards the origin will never be able to reach the origin, because it gets always reflected by the potential barrier. Therefore it can only probe the potential at an energy scale compatible with its initial energy. Perhaps a better analogy is given by a bound state electron within hydrogen. An electron in a hydrogen bound state cannot probe the origin of the attractive Coulomb potential. Normalizability gives a vanishing probability for such an electron to be located at the origin. This has nothing to do with Heisenberg uncertainty, because uncertainty relations hold only for normalizable wave functions, and so normalizability has to be imposed first, which proves to be sufficient to guarantee a vanishing probability measure at the origin.

Describing geodesic borders or their neighborhood in terms of observables requires to evolve fields from an initial surface towards the border. The evolution is not governed by a one-parameter group of isometric operators, but instead by a semigroup. As a consequence, norms of quantum states are not conserved, whereby quantum states refer to wave functionals over the space of field configurations in the Schrödinger representaton of quantum field theory. Semigroups appear here naturally since the spacetime is assumed to be absolutely inert against quantum fluctuations. Any frictionlike phenomena caused by this background reduces the state norm. Albeit the dynamics are not given by a unitary evolution, a probabilistic interpretation of the wave functional is still possible. The situation is analogous to the treatment of open quantum systems. This implies, however, that the spectrum of the Schrödinger Hamiltonian is not essentially self-adjoint. Of course, there is nothing wrong with this: Damping phenomena give rise to complex dispersion relations with a finite imaginary contribution. It is still possible to think of Hamiltonians as evolution generators in the usual infinitesimal sense. The pair (unitary, selfadjoint) is superseded by (contractive, accretive) in the spirit of Stone's theorem. This is the reason why it is convenient to consider evolution semigroups. In fact, there is no alternative: The geodesic borders considered here enforce a contractive evolution although the quantum fields become free in their vicinity. Even in this situation, the Hamiltonian is not a symmetry generator simply because the evolution is considered in an approximate asymptotic Kasner spacetime. 
So Bianchi type-I cosmologies are quantum complete and geodesic incomplete. This apparent clash of completeness concepts requires a resolution. The resolution is straightforward: Singularity diagnostics is a wellestablished method directly related to the mathematical spacetime model. It is based on the geodesic motion of point particles. Its physical realization implies an event set described by classical point particle phenomenology. This phenomenology is invalid close to spacelike geodesic borders where absorption and emission processes are operative. Classical particle phenomenology gives the wrong description of the observable events close to the geodesic border, and therefore it gives the wrong description of the physical spacetime bordering on spacelike singularities. An adequate characterization of the physical situation at the border requires quantum field theory. Once this is established, statements referring to Bianchi type-I cosmologies or their asymptotic Kasner approximations as geodesic incomplete are mathematically correct but physically irrelevant. Therefore it would be appropriate to call Bianchi type-I cosmologies just complete.

We have shown that Bianchi type-I cosmologies with approximate Kasner geometries close to their geodesic border and inflationary spacetimes away from the borders are quantum complete realizations of inflation. The argument is completely universal and holds for any inflaton potential. This is a reflection of the famous conjecture by Belinskii, Khalatnikhov and Lifshitz: In approximate Kasner regions, temporal changes dominate over any spatial correlations, and hence the inflaton evolution becomes effectively free, which is why these types of geodesic borders allow universal statements.

\section{ACKNOWLEDGMENTS}

It is a great pleasure to thank Abhay Ashtekar, Eugenio Bianchi, Kristina Giesel, Patrick Hager, Maximilian Kögler, Florian Niedermann, and Graham Shore for inspiring discussions. We appreciate financial support of our work by the DFG cluster of excellence "Origin and Structure of the Universe," TRR 33 "The Dark Universe," and the Alexander von Humboldt Foundation.

\section{APPENDIX: TEMPORAL GRADIENTS AND KASNER GEOMETRIES}

The conjecture of Belinskii, Khalatnikov and Lifshitz (BKL) stipulates that temporal gradients dominate over spatial gradients in the vicinity of a spacelike singularity. We present a quick argument that this guarantees Kasner geometries close to the geodesic border.

In a Gaussian normal neighborhood, $g=-\mathrm{d} t \otimes \mathrm{d} t+h$ and the BKL conjecture amounts to requiring $\left|\partial_{t} h\right| \gg\left|\partial_{x} h\right|$. The Levi-Civita connection is given by

$$
\Gamma_{a b}^{t}=\frac{1}{2} \partial_{t} h_{a b}+\mathcal{O}(\varepsilon), \quad \Gamma_{b t}^{a}=\frac{1}{2} g^{a c} \partial_{t} h_{c b}+\mathcal{O}(\varepsilon) .
$$

Here $a, b, c$ are spatial indices and all other Christoffel symbols are $\mathcal{O}(\varepsilon)$, where $\varepsilon:=\left|\partial_{x} h\right| /\left|\partial_{t} h\right|$ serves as the smallness parameter. Note that $\Gamma_{a b}^{t}$ are the extrinsic curvature components $K_{a b}$ in this coordinate neighborhood. Therefore, up to $\mathcal{O}(\varepsilon)$,

$$
\begin{aligned}
& (\mathrm{Ric})_{t t}=-\partial_{t} \operatorname{Tr}_{h}(K)-\operatorname{Tr}_{h}(K K), \\
& (\text { Ric })_{a b}=\partial_{t} K_{a b}-(K K)_{a b},
\end{aligned}
$$

with $(K K)_{a b}:=K_{a c} h^{c d} K_{d b}$. Note that $(\mathrm{Ric})_{t a}=\mathcal{O}(\varepsilon)$. This poses no problem provided $h$ is diagonal since then $(\text { Ric })_{t a}=0$. Assuming $h$ is diagonal, $h_{a c}=\delta_{a \hat{c}} w_{\hat{c}}$, the extrinsic curvature becomes $K_{a c}=\delta_{a \hat{c}} \dot{w}_{\hat{c}} / 2$, where hats over spatial indices suspend the usual summation convention, and overdots denote time derivatives. For a vacuum solution,

$$
(\text { Ric })_{t t}=\frac{1}{2} \sum_{a=1}^{3}\left[\frac{\ddot{w}_{a}}{w_{a}}-\frac{1}{2}\left(\frac{\dot{w}_{a}}{w_{a}}\right)^{2}\right]=0 \text {. }
$$

This is solved by $w_{a}=t^{2 p_{a}}$, provided $p_{1}+p_{2}+p_{3}=$ $p_{1}^{2}+p_{2}^{2}+p_{3}^{2}$, which is precisely the Kasner condition.
[1] A. H. Guth, Phys. Rev. D 23, 347 (1981).

[2] A. D. Linde, Phys. Lett. B 108, 389 (1982).

[3] A. Albrecht and P. J. Steinhardt, Phys. Rev. Lett. 48, 1220 (1982).

[4] T. S. Pereira, C. Pitrou, and J.-P. Uzan, J. Cosmol. Astropart. Phys. 09 (2007) 006.

[5] A. Ashtekar, A. Henderson, and D. Sloan, Phys. Rev. D 83, 084024 (2011).
[6] T. Damour, M. Henneaux, and H. Nicolai, Classical Quantum Gravity 20. R145 (2003).

[7] A. Ashtekar and E. Wilson-Ewing, Phys. Rev. D 79, 083535 (2009).

[8] S. W. Hawking and R. Penrose, Proc. R. Soc. A 314, 529 (1970).

[9] I. Agullo and A. Ashtekar, Phys. Rev. D 91, 124010 (2015). 
[10] J. Feldbrugge, J.-L. Lehners, and N. Turok, Phys. Rev. Lett. 119, 171301 (2017).

[11] V. A. Belinski, E. Lifshitz, and I. Khalatnikov, Sov. Phys. 13, 745 (1971).

[12] V. A. Belinski, I. Khalatnikov, and E. Lifshitz, Adv. Phys. 31, 639 (1982).

[13] A. Ashtekar, A. Henderson, and D. Sloan, Classical Quantum Gravity 26, 052001 (2009).

[14] S. Hofmann and M. Schneider, Phys. Rev. D 91, 125028 (2015).
[15] L. Kofman, J.-P. Uzan, and C. Pitrou, J. Cosmol. Astropart. Phys. 05 (2011) 011.

[16] A. E. Gümrükçüoglu, L. Kofman, and M. Peloso, Phys. Rev. D 78, 103525 (2008).

[17] A. E. Gümrükçüoglu, C. R. Contaldi, and M. Peloso, J. Cosmol. Astropart. Phys. 11 (2007) 005.

[18] S. Gielen and N. Turok, Phys. Rev. Lett. 117, 021301 (2016).

[19] S. Hofmann and M. Schneider, Phys. Rev. D 95, 065033 (2017). 